\title{
The Delamination Study of Laminated Composites with XFEM/VCCT
}

\author{
Xin Xiao * \\ School of Astronautics, Beihang University, Beijing 100191, PR China \\ E-mail: xiaoxin@buaa.edu.cn
}

Keywords: the extended finite element method (XFEM); Virtual Crack Closure Technology (VCCT); composites; delamination; laminate

\begin{abstract}
The extended finite element method (XFEM) is an effective way to solve the fracture problem. This paper introduced a method combined VCCT with extended finite element method to realize the numerical simulation of composite materials delamination. The process of delamination of single crack and multiple cracks in unidirectional laminate were simulated and revealed two cracks propagation mechanisms further. Numerical results have a good comparison with the experiment results. Studies show that the XFEM/VCCT method can effectively simulate the delamination process of composites laminates.
\end{abstract}

\section{Introduction}

Carbon fiber composites have the advantages of high designability, high specific strength and modulus which make it widely used in aerospace and other fields ${ }^{[1-3]}$. Theoretical analyses and experimental studies in recent years have shown that the weak parts of laminated composites are between layers, and the delamination is the main form of destruction in laminated composites engineering applications ${ }^{[4,5]}$. Delamination which is concealed in composite laminate structures is a security issue that cannot be ignored. Therefore, it is of great engineering significance to use the numerical simulation method to study the mechanism and regularity of the delamination of composite materials ${ }^{[6]}$.

The delamination essentially belongs to a kind of material fracture phenomenon, which is often analyzed by the fracture mechanics method ${ }^{[5]}$. Virtual Crack Closure Technology (VCCT) and Cohesive Force Simulation (CZM), both of which have been widely used to simulate delamination of unidirectional laminates. However, VCCT and CZM have one obvious disadvantage that they all need to predetermine the path that the crack may expand, and use the traditional finite element method to calculate the crack propagation. In order to obtain sufficient calculation accuracy, the grid needs to be subdivided enough and the calculation is complicated and enormous. The extended finite element method (XFEM) ${ }^{[7,8]}$ can alleviate the shortcomings of the crack surface meshing. The scholars at home and abroad combined with CZM have verified the feasibility of the XFEM delamination simulation for composite laminates ${ }^{[9,10]}$.

Van der Meer et al. ${ }^{[11]}$ believe that for large-scale deformations, the focus is on the total energy dissipated during the crack propagation process rather than just the cohesion region. Therefore, 
VCCT is used instead of the crack tip CZM to simulate the crack propagation, thus avoiding crack tip size constraints. VCCT ${ }^{[12,13]}$ has the advantages of simple solution, high precision, easy calculation of energy release rate, and low requirements on grid size. In this paper, the virtual crack closure technique is combined with the extended finite element method to analyze the delamination extension of laminates.

\section{XFEMVCCT-based Delamination Model for Composites}

\subsection{The basic principle of XFEM}

The extended finite element method (XFEM) is a special case of the local decomposition of the unit decomposition method and the generalized finite element method. The approximate field around the crack based on the extended finite element method ${ }^{[14]}$ is

$$
u^{h}(\mathrm{x})=\sum_{I \in \mathbb{N}} N_{I}(\mathrm{x}) u_{I}+\sum_{I \in \aleph_{\Gamma}} N_{I}(\mathrm{x}) H(\mathrm{x}) a_{I}+\sum_{I \in \aleph_{\Lambda}} N_{I}(\mathrm{x}) \sum_{\alpha=1}^{n} F_{\alpha}(\mathrm{x}) b_{I}^{\alpha}
$$

Where $u_{I}$ is the nodal displacement vector associated with the continuous part of the finite element solution, $a_{I}$ is the node expansion degree associated with the Heaviside function, $b_{I}^{\alpha}$ is the node expansion degree of freedom associated with the elastic progressive crack field function $F_{\alpha}(\mathrm{x})$. Different forms of crack tip asymptotic functions are related to crack position and degree of deformation of nonlinear material. $\aleph 、 \aleph_{\Gamma} 、 \aleph_{\Lambda}$ refer to the set of all nodes, the set of nodes whose shape function domain is split by a crack (not including crack tip), and the set of nodes whose shape function domain is split by a split tip.

Heaviside function $H(x)$ : at the top of the crack, the value is taken as 1 ; at the bottom of the crack, the value is taken as -1 which can be written as

$$
H(x)= \begin{cases}1 & \left(x-x^{*}\right) \cdot e_{n}>0 \\ -1 & \left(x-x^{*}\right) \cdot e_{n}<0\end{cases}
$$

Where $X$ is the point of investigation, $\boldsymbol{x}^{*}$ is the nearest point to $\boldsymbol{X}$ near the crack, and $\boldsymbol{e}_{\boldsymbol{n}}$ is the unit normal vector outside the crack at $\boldsymbol{x}^{*}$.

XFEM provides great flexibility in the construction of shape functions and approximate space. The introduction of extension functions such as Heaviside function and elastic progressive crack tip function enables XFEM to simulate displacement field discontinuities caused by cracks and singularities of crack tip stress fields. The problem is that the boundary of the element does not have to be consistent with the geometry of the crack, and the crack tip element does not need to be overly subdivided. At the same time, various problems brought about by the repartitioning of the mesh are avoided in the problem of crack propagation.

In Abaqus/Standard, singular asymptotic functions are only used when establishing fixed crack models. For the problem of crack propagation, the method of combining XFEM and virtual crack closure is used in this paper to simulate crack propagation.

\subsection{XFEM-VCCT Crack Propagation Model}

This article is aimed at the simulation of the delamination behavior of composite materials. The 
establishment of the model is based on the VCCT method. For this purpose, the fracture criterion and the crack propagation path must be defined.

\subsubsection{Fracture criterion}

The fracture criterion serves as a criterion for crack propagation and determines when the crack starts to crack. The premise of crack propagation is that the total strain energy release rate satisfying the fracture condition is greater than the critical energy release rate.

In this paper, the B-K criterion considering the stratified hybrid model is used as the fracture criterion

$$
G_{\text {IC }}+\left(G_{\text {IIC }}-G_{\text {IC }}\right) \beta^{\eta}=G
$$

Where $G_{\mathrm{IC}}, G_{\mathrm{IIC}}$ and $\eta$ are the specified material parameters respectively, $\beta=G_{\mathrm{II}} /\left(G_{\mathrm{I}}+G_{\mathrm{II}}\right)$.

For the fracture criterion, the critical energy release rate $G$ must be solved by the virtual crack closure method in Abaqus. In the Abaqus environment, the XFEM-VCCT method superimposes the virtual nodes on the initial real nodes by introducing virtual nodes to represent the discontinuity of the broken units, as shown in Fig. 1. When the unit remains intact, each virtual node is constrained to the corresponding real node. The use of a virtual node is used to indicate that the unit breaks when the fracture criterion is met, which means the strain energy release rate at the crack tip exceeds the fracture toughness of the material.

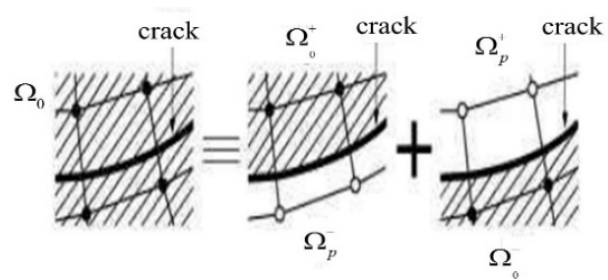

Fig 1. Virtual Nodes Schematic

The basic idea of the virtual crack closure technique is to assume that the amount of energy released by a trace of crack propagation is equal to the work done by closing the crack to its original length. Therefore, the strain energy release rate $G^{[15]}$ can be calculated from the stress values and displacement values of the crack propagation and type I and type II.

\subsubsection{Extended path design}

In the Abaqus finite element software environment, there are three forms of crack propagation in the extended finite element method based on VCCT, parallel to the material 1 direction, perpendicular to the material 1 direction, and the direction of the maximum shear stress.

For DCB specimens under type I loading, crack propagation occurs along the direction of the fiber lay-up. Therefore, the simulation is performed in the form of a crack propagation path parallel to the material and set in one direction.

\section{Typical delamination examples}

In order to verify the proposed composite finite element method combined with the virtual crack closure technique for the delamination of composite laminates, the unilateral laminate single layer was first discussed because the standard test method for these interface fracture properties is the starting point for the study of all the stratification problems; then the more general problem, the type I loading process of a unidirectional laminate containing two delaminated cracks was analyzed. 


\subsection{Single delamination in unidirectional laminate}

The type I stratification test was conducted using the test in [16], which was conducted in accordance with ASTM D 5528-01. The configuration and geometric dimensions of a DCB (Double Cantilever Beam) test piece are shown in Fig 2(a) where $h$ is the thickness of the upper arm or lower arm of the test piece, $B$ represents the width of the test piece, and the average single layer thickness of the composite material is $0.185 \mathrm{~mm}$. The loading diagram is shown in Figure 2(b). In order to obtain an initial layer length $\mathrm{a}_{0}$ of $40 \mathrm{~mm}$, a $40 \mathrm{~mm}$ long PTFE film was embedded in one side of the predetermined layer as a buried delamination.

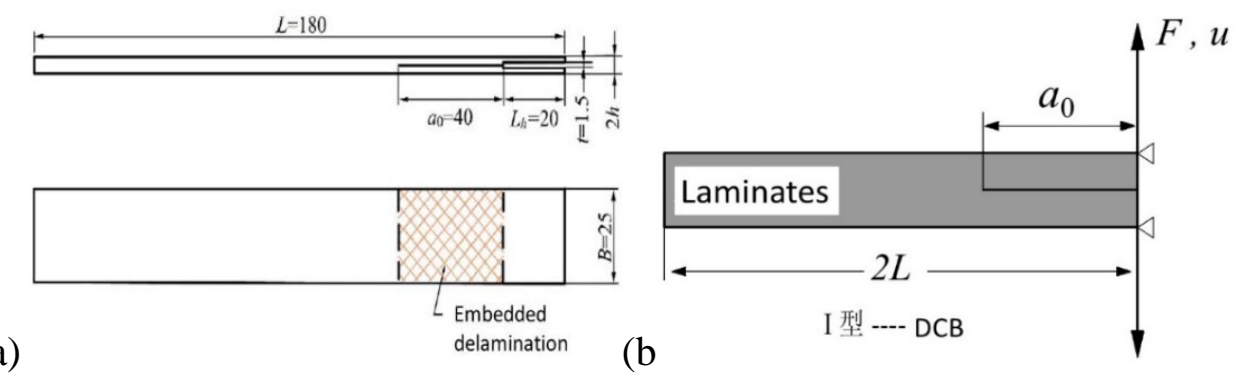

Fig 2. DCB Specimen configuration and Geometry loading diagrams

Plane strain cell CPE4 is used to simulate unidirectional laminates, and uniform mesh division of $0.3 \mathrm{~mm}$ is adopted to meet the solution accuracy. The connection between the laminate and the beam is achieved by dynamic constraints that correspond to the two translational degrees of freedom of the node. The initial crack location is indicated by a $40 \mathrm{~mm}$ long straight line in the laminate part. In order to obtain a stable crack growth, the specimen is loaded by displacement control. The numerical model is shown in Fig. 3.

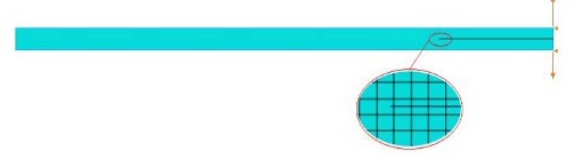

Fig 3. DCB numerical model

\subsection{Multi-delamination in unidirectional laminates}

Standard tests tested the interfacial properties of the composite under different fracture modes for a single interface crack. However, in practical problems, there are often two or more interface delamination inside the laminate, such as laminates after low-velocity impact. Therefore, the example in [17] was used to further confirm the ability of XFEM to predict mixed-mode multi-stratification in unidirectional laminates. The geometry of the specimen is shown in Fig 4. Two initial cracks are preset between different pairs of plies. The laminate is HTA913 carbon-resin composite material and its properties are as follows: $E_{11}=126 \mathrm{GPa}, E_{22}=8.5 \mathrm{GPa}, G_{12}=4.5 \mathrm{GPa}$, $v_{12}=0.29, t_{n}^{0}=3.3 \mathrm{MPa}, t_{s}^{0}=7.0 \mathrm{MPa}, G_{\mathrm{I}}^{\mathrm{C}}=330 \mathrm{Nm}^{-1}, G_{\mathrm{II}}^{\mathrm{C}}=800 \mathrm{Nm}^{-1}$.

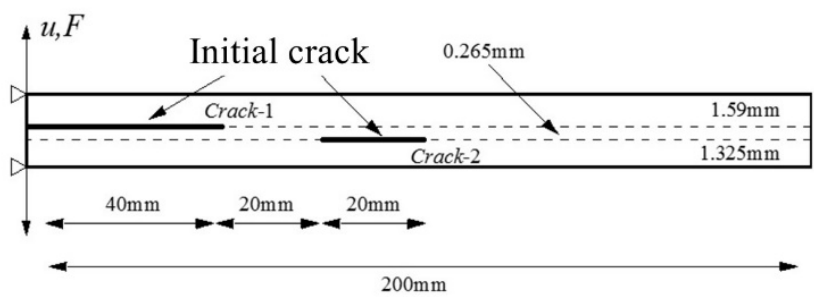

Fig 4. Geometric parameters and loading forms of multi-delamination specimens 
Similar to a single-delamination test piece, the numerical model of a unidirectional laminate multi-layer test piece is given by a $0.3 \mathrm{~mm}$ wide plane strain element.

\section{Results and Discussion}

\subsection{Influence of Grid Density}

For the DCB model, a plane strain cell CPE4 was used to design four groups of grids with 0.1 $\mathrm{mm}, 0.3 \mathrm{~mm}, 1 \mathrm{~mm}$, and $3 \mathrm{~mm}$ length along the crack side of the cell, and the load displacement relationship is shown in Fig 5.

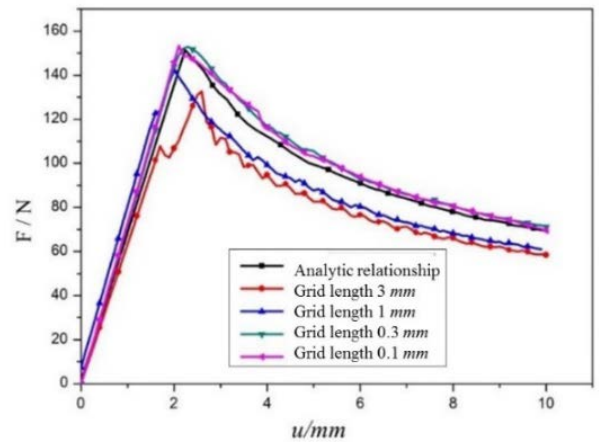

Fig 5. Load-displacement relationship of different grid density

From Fig 5, it can be seen that under the four kinds of grid densities, the load-displacement relationship is consistent with the experimental results. The higher the density, the closer the numerical results to the experimental results. When the grid side lengths are $0.3 \mathrm{~mm}$ and $0.1 \mathrm{~mm}$, respectively, the number of grids is 11193 and 33579, and the critical load change is very small, which indicates that the number of grids can meet the design accuracy requirement. Therefore, in the engineering calculation, when using this method for calculation and analysis, qualitative analysis can be performed with fewer grids, and then the calculation of grid density can be appropriately increased. Therefore, the subsequent calculation and analysis use the grid length of $0.3 \mathrm{~mm}$.

\subsection{Single delamination in unidirectional laminate}

(a)

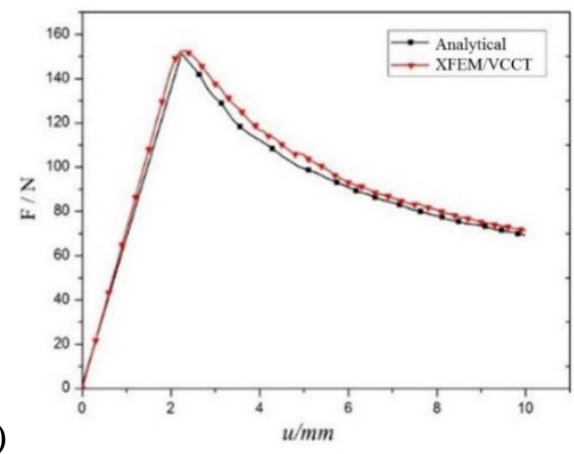

(b)

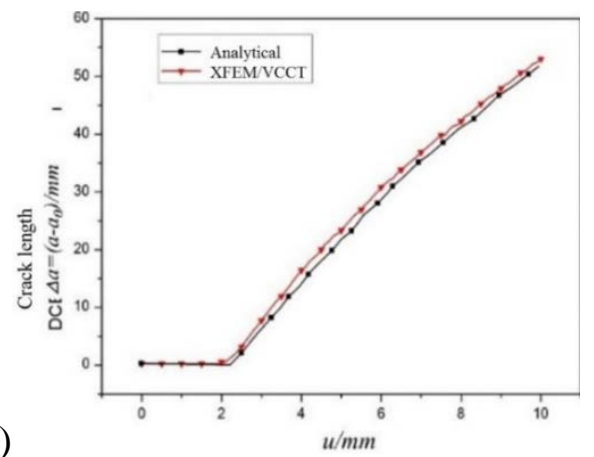

Fig 6. DCB numerical simulation results(a) Load displacement relationship (b) Crack propagation

DCB test pieces, Fig 6 shows the load displacement relationship and crack growth. As the displacement increases, the load first increases linearly. Then the stiffness begins to drop, and the load-displacement curve quickly reaches its extreme value. After the extremes, the load gradually decreases. Correspondingly, cracks expand at a certain rate. Fig 6(b) shows the crack propagation 
state simulated by XFEM. It can be seen that the crack splits the element into two parts in the open mode. The results show that the results obtained by numerical analysis using XFEM/VCCT are in good agreement with the analytical results, which verifies the feasibility of the method

\subsection{Multi-delamination in unidirectional laminates}

Fig 7 compares the numerical results of the XFEM simulation with the results of the Alfano experiment [17]. It can be seen that the loading process includes five stages: 1) In the O-A stage, as the displacement increases, the stiffness of the load displacement curve gradually decreases. 2) After point A, crack 1 expands more rapidly than O-A section. 3) In the B-C phase, a significant load drop can be observed in Fig. 7(a). In addition, crack 1 will also suddenly jump. 4) In the C-D phase, two cracks slowly expand and the load maintains a steady upward trend. 5) After point D, both cracks will expand rapidly. Overall, the XFEM simulated load displacement results agree well with the experimental results and also the literature [10]. This also confirms that XFEM/VCCT simulation can accurately predict the mechanical behavior of layered laminates and reveal the mechanism of crack propagation.

(a)
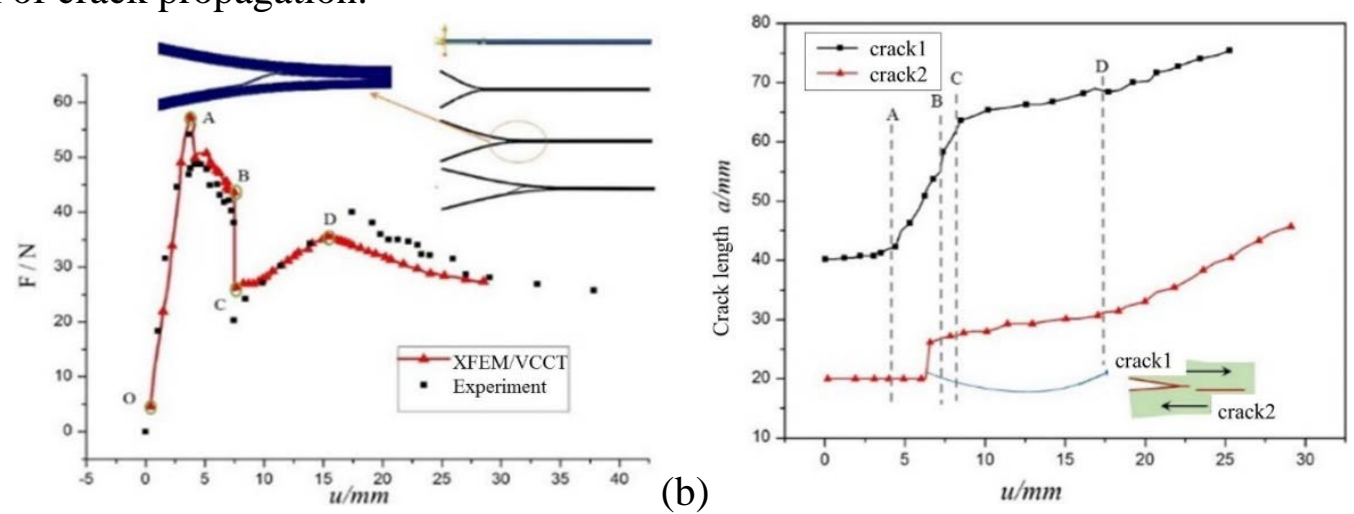

Fig.7 Numerical results of multi-delamination specimens of unidirectional laminates (a) Load displacement curve (b) Crack propagation XFEM simulation results

\section{Conclusion}

This paper introduces the basic principles of the combined application of extended finite element and virtual crack closure techniques. XFEM uses the concept of global partitioning to facilitate the insertion of extended functions into finite element approximations. The discontinuity of cracks can be exploited by extension functions associated with additional degrees of freedom to confirm. By introducing a virtual node and using the VCCT principle to calculate the strain energy release rate, the crack can be extended independently of the grid. This paper attempts to use this method to simulate the single crack propagation process and double crack propagation of composite unidirectional laminates. The results of the study visually give the entire process of crack propagation of laminates.

1) The numerical results are in good agreement with the analytical results and the test results. It is proved that it is feasible to use XFEM/VCCT to simulate the delamination process of laminates and study its macroscopic mechanical properties;

2) Using this method for numerical analysis, proper density of grid seed placement can obtain more accurate numerical results and save computational cost;

3) In the DCB test, the load of the single-ply delamination specimen first increases linearly, then the stiffness begins to decrease, and the load-displacement curve quickly reaches its extremum. After the extremum passes, the load gradually decreases. Correspondingly, the crack is at a certain 
rate to develop;

4) The numerical load displacement curves of the multi-delamination specimens of type I-dominated unidirectional laminates are in good agreement with the experimental results, and the two crack propagation mechanisms are revealed by XFEM/VCCT.

Of course, the work in this paper is only a tentative exploration, and more in-depth research is needed, such as the expansion of multi-directional laminates, and the use of this method to study energy release rates.

\section{References}

[1] Liu P F, Zheng J Y. Recent developments on damage modeling and finite element analysis for composite laminates; A review[J]. Materials \& Design, 2010,31(8);3825-3834.

[2] Xing L, Bao J, Songming L I, et al. Development status and facing challenge of advanced polymer matrix composites[J]. Acta Materiae Compositae Sinica, 2016.

[3] Tang J. Development Status and Prospects of Aerospace Composite Materials [J]. Spacecraft Environment Engineering,2013,30(04):352-359.

[4] Qu P. Influence of Multi-scale Structures of Fiber Reinforce Resin Matix Composites on Mechanical Properties [D]. Shandong University,2012.

[5] Yao L J. Mode I quasi-static and fatigue delamination growth in composite laminates [D]. Northwestern Polytechnical University,2016.

[6] Yazdani S, Rust W J H, Wriggers P. An XFEM approach for modelling delamination in composite laminates[J]. Composite Structures, 2016, 135:353-364.

[7] Di Y L, Wang H D, Dong L H, et al. Application of the Extended Finite Element Method in Crack Propagation [J]. Material Guide,2017,31(03):70-74+85.

[8] Su Y, Wang S N, Gao W. Generalized Extended Finite Element Method and Its Application in Crack Growth [J]. Journal of Northwestern Polytechnical University,2015,33(06):921-927.

[9] T.E. Tay, X.S. Sun, V.B.C. Tan. Recent efforts towards modeling interaction of matrix cracks and delaminations: an integrated XFEM-CE approach[J]. Advanced Composite Materials. 2014. 23:391-408.

[10] Zhao L, Zhi J, Zhang J, et al. XFEM simulation of delamination in composite laminates[J]. Composites Part A Applied Science \& Manufacturing, 2016, 80:61-71.

[11] Van Der Meer F. P., Moës N., Sluys L. J. A level set model for delamination--Modeling crack growth without cohesive zone or stress singularity[J]. Engineering Fracture Mechanics. 2012. 79: 191-212.

[12] Zhang Y H, Zhou L M, Tian W J. Node-Based Smoothed FEM for Virtual Crack Closure Technique [J]. Quarterly Journal of Mechanics,2015,36(01):134-140.

[13] Ma C W, Li Z G, Lu G F, et al. Development on Delamiantion Characteristics of Integrated Composite Structures: Part II[J]. Aircraft design,2014,34(06):32-41.

[14] Belytschko T., Black T. Elastic crack growth in finite elements with minimal remeshing[J]. International Journal for Numerical Methods in Engineering, 1999, 45(5): 601-620.

[15] Sang Y C. Application of CZM and VCCT in Numerical Simulation of Crack Propagation [D]. Lanzhou University of Technology,2017.

[16] Wang Y N. Study on Mechanism of Delamination Growth Resistance in Multidirectional Composites Laminates [D]. Beihang University,2017.

[17] Alfano G., Crisfield M. A. Finite element interface models for the delamination analysis of laminated composites: mechanical and computational issues[J]. International Journal for Numerical Methods in Engineering, 2001, 50(7): 1701-1736. 